Homology, Homotopy and Applications, vol.16(1), 2014, pp.295-311

\title{
GRAPHS ASSOCIATED WITH SIMPLICIAL COMPLEXES
}

\author{
A. GRIGOR'YAN, YU.V. MURANOV AND SHING-TUNG YAU
}

(communicated by Alexander Mishchenko)

\begin{abstract}
The cohomology of digraphs was introduced for the first time by Dimakis and Müller-Hoissen. Their algebraic definition is based on a differential calculus on an algebra of functions on the set of vertices with relations that follow naturally from the structure of the set of edges. A dual notion of homology of digraphs, based on the notion of path complex, was introduced by the authors, and the first methods for computing the (co)homology groups were developed. The interest in homology on digraphs is motivated by physical applications and relations between algebraic and geometrical properties of quivers. The digraph $G_{B}$ of the partially ordered set $B_{S}$ of simplexes of a simplicial complex $S$ has graph homology that is isomorphic to the simplicial homology of $S$. In this paper, we introduce the concept of cubical digraphs and describe their homology properties. In particular, we define a cubical subgraph $G_{S}$ of $G_{B}$, whose homologies are isomorphic to the simplicial homologies of $S$.
\end{abstract}

\section{Introduction}

In a recent paper $[\mathbf{9}]$, the authors developed the theory of homology of path complexes, which can be considered as a natural generalization of a simplicial homology theory (see, for example, $[\mathbf{1 0}],[\mathbf{1 1}]$, and $[\mathbf{1 2}]$ ). This approach allows us to define the notion of homology for digraphs that is dual to the notion of cohomology of $[\mathbf{2}],[\mathbf{3}]$, and $[8]$.

Any graph can be naturally regarded as a 1-dimensional simplicial complex, so that its simplicial homologies of all dimensions $n \geqslant 2$ are trivial. However, as was shown in [9] on many examples, the graph homologies of a digraph can be highly non-trivial for any $n$, as this theory detects automatically higher-dimensional substructures of the digraph; for example, a graphical simplex or cube with an appropriate direction of edges.

Generally speaking, a digraph $G$ can be turned into a simplicial complex $S$ in many ways, by constructing higher-dimensional simplexes on some of its cliques (a clique

Received July 3, 2013, revised December 13, 2013; published on May 28, 2014.

2010 Mathematics Subject Classification: 05C25, 18G60, 52C99, 55U10, 57M15.

Key words and phrases: (co)homology of digraphs, path complex of a digraph, simplicial homology, cubical digraph, cubical complex, simplicial complex.

Article available at http://dx.doi.org/10.4310/HHA.2014.v16.n1.a16

Copyright (C) 2014, International Press. Permission to copy for private use granted. 
in a graph is a subset of its vertices such that every pair of vertices in the subset is connected by an (undirected) edge). These, however, do not have to match the higher dimensional substructures of $G$ that are predetermined by $G$ (see, for example, $[\mathbf{1}]$ and $[\mathbf{7}])$.

On the other hand, any simplicial complex $S$ naturally determines an (undirected) graph $S_{1}$ that is the 1-skeleton of $S$. The graph $S_{1}$ can be turned into a digraph by choosing arbitrary directions of the edges. Simple examples show that the simplicial homologies of $S$ and the graph homologies of $S_{1}$ can be different regardless of the choice of the digraph structure on $S_{1}$ (see example in Section 3).

Now let $S$ be a finite simplicial complex and let $B_{S}$ be the set of its simplexes. Consider a graph $G_{B}$ with vertex set $B_{S}$ and an arrow $\sigma \rightarrow \tau$ if and only if ( $\tau \subset$ $\sigma) \&(\tau \neq \sigma)$. Then the dual chain complex to the complex for the graph cohomology of $S_{B}$ is isomorphic to the simplicial chain complex of the first barycentric subdivision of $S$ (see [8]).

Let $G_{S}$ be a subgraph of $G_{B}$, with the same set of vertices $B_{S}$, and with $s, t \in B_{S}$ connected in $G_{S}$ by a directed edge $s \rightarrow t$ if and only if

$$
s \supset t \text { and } \operatorname{dim} s=\operatorname{dim} t+1 .
$$

The graph $G_{S}$ can be realized geometrically as follows. Denote by $b_{s}$ the barycenter of a simplex $s \in S$. Then the set $B_{S}$ coincides with the set of barycenters of all $s \in S$. Define the edges $b_{s} \rightarrow b_{t}$ between two barycenters by the same rule (1); this gives a digraph $G_{B}$ (see Fig. $1(b)$ ).
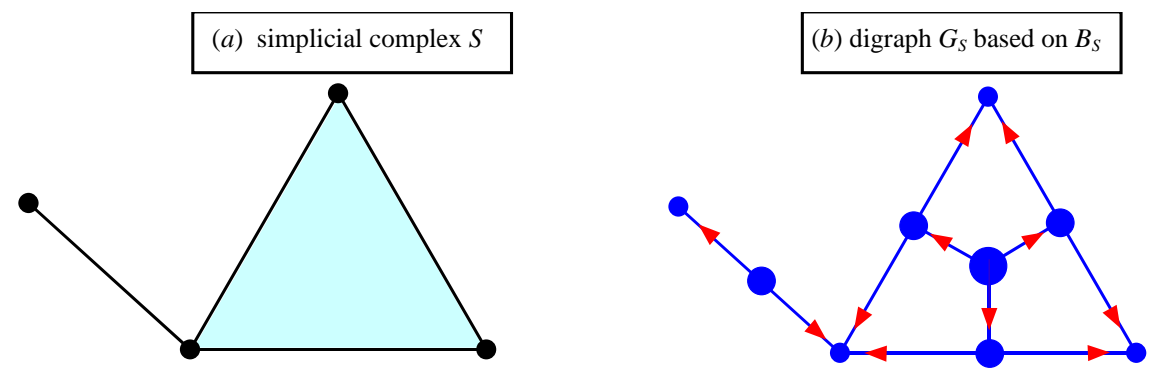

$(c)$ abstract digraph $G_{S}$

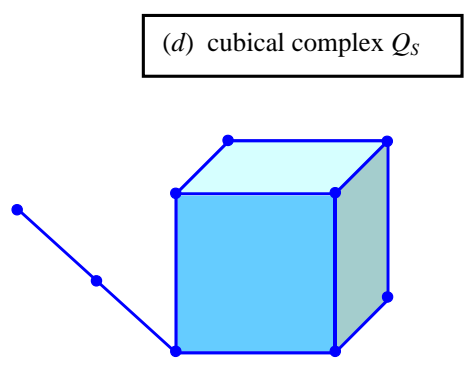

Figure 1: A simplicial complex $S$, the digraph $G_{S}$ realized on the barycenters, and, abstractly, and the cubical complex $Q_{S}$. 
Furthermore, it is not difficult to see that $G_{S}$ is the 1-skeleton of a natural cubical complex associated with $S$, which will be denoted by $Q_{S}$. More precisely, $Q_{S}$ can be constructed as follows. For each simplex $s \in S$, consider a full barycentric subdivision $s^{b}$ of $s$, and for any vertex $v$ of $s$ take the union of all the elements of $s^{b}$ containing $v$. This union is a topological cube, and the family of all such cubes of all simplexes $s \in S$ forms a cubical complex $Q_{S}$ that is a cubillage of $S$ (cf. $[\mathbf{5}, \S 5]$ ). Thus we obtain a new relation between graph homologies and cubical lattices of topological spaces (see [4] and [5] for physical applications of cubical lattices).

The complexes $S$ and $Q_{S}$ have the same topological realization, which implies that their cell homologies are the same. On the other hand, we prove in Section 5 that the cell homology chain complex of $Q_{S}$ and the graph homology chain complex of $G_{S}$ are isomorphic, which implies the isomorphism of $H_{*}(S)$ and $H_{*}\left(G_{S}\right)$. In particular, this approach provides the possibility of computing homologies of complicated cubical digraphs.

It is worth mentioning that the assignment $S \mapsto G_{S}$ is a functor from the category of simplicial complexes with inclusion maps to the category of digraphs with inclusion maps.

In Section 2, we give necessary preliminary material about simplicial and cubical complexes and their homology properties, following $[\mathbf{6}]$, [10], and [12]. In particular, we discuss in detail the procedure for constructing of the cubical complex $Q_{S}$ mentioned above. In Section 3 we give a brief account of the graph homology theory following [9]. In Section 4, we define cubical digraphs and describe theirs properties. Finally, in Section 5 we prove the main result, Theorem 5.1.

\section{Acknowledgments}

The authors thank the grant "Geometry and Topology of Complex Networks," no. FA-9550-13-1-0097, for support. The first and second authors were supported in part by SFB 701 of German Research Council. The second author was supported in part by Conacyt Grants 98697 and 151338. We thank the referees for helpful comments that improved the paper.

\section{Simplicial and cubical complexes}

In this section we present necessary material about simplicial and cubical complexes and describe the construction of a cubical complex associated with a given simplicial complex. The details can be found in [6] and [12].

By an $n$-dimensional simplex we mean a non-degenerate affine image of the standard simplex

$$
\Delta^{n}=\left\{\left(x_{0}, x_{1}, \ldots, x_{n}\right) \in \mathbb{R}^{n+1}: x_{0}+x_{1}+\cdots+x_{n}=1, x_{i} \geqslant 0 \text { for all } i=0, \ldots, n\right\}
$$

in some space $\mathbb{R}^{N}$. Recall that a finite simplicial complex $S$ is a finite family of simplexes in $\mathbb{R}^{N}$ such that the following conditions are satisfied:

1. if $S$ contains a simplex $s$ then $S$ contains all the faces ${ }^{1}$ of $s$;

\footnotetext{
${ }^{1}$ Contrary to a common convention, we do not regard $\emptyset$ as a face.
} 
2. if $s_{1}, s_{2}$ are two simplexes from $S$ then the intersection $s_{1} \cap s_{2}$ is either empty or a simplex from $S$.

Let us describe the lesser known notion of a cubical complex. The standard $n$ dimensional cube $I^{n}$ is defined for $n \geqslant 1$ by:

$$
I^{n}=\left\{\left(x_{1}, \ldots, x_{n}\right) \in \mathbb{R}^{n}: 0 \leqslant x_{i} \leqslant 1, i=1, \ldots, n\right\},
$$

and for $n=0$ by $I^{0}=\{0\}$. An $n$-dimensional cube $q$ is a non-degenerate piecewise linear image of $I^{n}$ in some $\mathbb{R}^{N}$. We would like to point out that in opposition to the definition of a simplex, we use here a piecewise linear image of a standard cube.

A $k$-dimensional face of $I^{n}$ is any of the $k$-cubes

$$
\left\{\left(x_{1}, \ldots, x_{n}\right) \in I^{n}: x_{i_{1}}=\varepsilon_{1}, \ldots, x_{i_{n-k}}=\varepsilon_{n-k}\right\}
$$

where $1 \leqslant i_{1}<\cdots<i_{n-k} \leqslant n$ and $\varepsilon_{j}=0$ or 1 , and a $k$-dimensional face of $q$ is the image under the same mapping $I^{n} \rightarrow \mathbb{R}^{N}$ of one of the $k$-dimensional faces of $I^{n}$.

A finite cubical complex $Q$ is a finite collections of cubes in some $\mathbb{R}^{N}$ such that

(i) if $Q$ contains a cube $q$ then $Q$ contains all the faces of $q$;

(ii) if $q_{1}, q_{2}$ are two cubes from $Q$ then the intersection $q_{1} \cap q_{2}$ is either empty or a cube from $Q$.

In this paper we will consider only finite simplicial and cubical complexes, so that the adjective "finite" will be omitted. Clearly, both simplicial and cubical complexes have an underlying structure of a topological space and even a structure of a polyhedron. Denote by $|S|$ the union of all simplexes from a simplicial complex $S$ and similarly by $|Q|$ - the union of all cubes from $Q$. Both $|S|$ and $|Q|$ will be regarded as topological spaces with the induced topology from the ambient space $\mathbb{R}^{N}$.

Fix a ring $\mathbb{K}$. Each simplicial complex $S$ gives rise to a chain complex $C_{*}(S)$ over $\mathbb{K}$ with a boundary operator $\partial$, and, hence, to the simplicial homologies $H_{*}\left(C_{*}(S)\right) \cong$ $H_{*}(|S|)$, and, similarly, one obtains a cubical chain complex $C_{*}(Q)$ over $\mathbb{K}$ with a boundary operator $\partial$ and the corresponding cubical homologies $H_{*}\left(C_{*}(Q)\right) \cong$ $H_{*}(|Q|)$, where $H_{*}(|S|)$ and $H_{*}(|Q|)$ are the singular homologies of the topological spaces $|S|$ and $|Q|$, respectively.

For any simplicial complex $S$, we will construct an associated cubical complex $Q_{S}$ with the same underlying topological space $|S|=\left|Q_{S}\right|$.

Denote by $S^{b}$ the barycentric subdivision of $S$. Now for any $k$-simplex $s \in S$ and a vertex $v$ of $s$, define a set $q_{s, v}$ by

$$
q_{s, v}=\bigcup_{\left\{t \in S^{b}: v \in t\right\}} t
$$

that is, $q_{s, v}$ is the union of all simplexes from $s^{b}$ that contain the vertex $v$. It is not difficult to see that $q_{s, v}$ is a $k$-cube (see [5] and [13] for the details). It is also clear that $s$ is the union of all the cubes $q_{s, v}$ over all vertices $v$ of $s$ (cf. Fig. 2).

The collection of all cubes $\left\{q_{s, v}\right\}$ over all $s \in S$ and $v \in s$ is then a cubical complex that will be denoted by $Q_{S}$. It is clear from the construction that $H_{*}\left(C_{*}(S)\right) \cong$ $H_{*}\left(C_{*}\left(Q_{S}\right)\right)$.

By construction, the set of vertices of $Q_{S}$ coincides with the set $B_{S}$ of the barycenters of all simplexes of $S$. The 1-dimensional skeleton of the cubical complex $Q_{S}$ can 


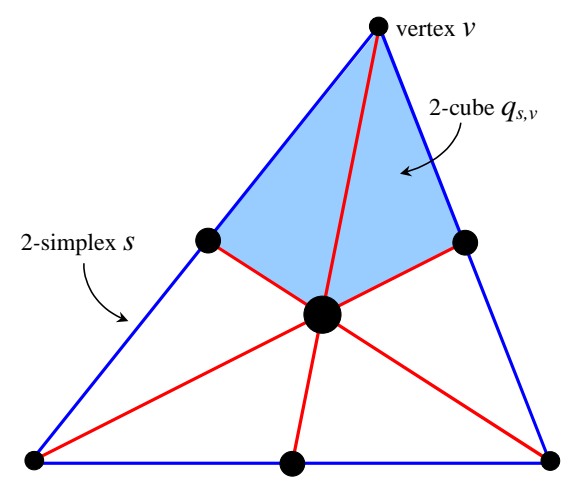

Figure 2: Construction of a cube $q_{s, v}$.

be described as follows. Given two simplexes $s, t$ of $S$, let us connect their barycenters $b_{s}$ and $b_{t}$ by a segment $\left[b_{s}, b_{t}\right]$ if and only if $s=t \cup\{v\}$ for some vertex $v \notin t$. Then the 1-dimensional skeleton of $Q_{S}$ is given by the union of all such segments $\left[b_{s}, b_{t}\right]$ (cf. Fig. 1).

\section{Homologies of digraphs}

In this section we cite necessary material from [9]. In this paper $\mathbb{K}$ is a fixed commutative ring with a unity 1.

Let $V$ be a finite set, whose elements will be called vertices. An elementary p-path on a finite set $V$ is any (ordered) sequence $i_{0}, \ldots, i_{p}$ of $p+1$ vertices of $V$, which will be denoted by $i_{0} \ldots i_{p}$ or by $e_{i_{0} \ldots i_{p}}$. Denote by $\Lambda_{p}=\Lambda_{p}(V)$ the free $\mathbb{K}$-module generated of all elementary $p$-paths $e_{i_{0} \ldots i_{p}}$ with coefficients from $\mathbb{K}$. The elements of $\Lambda_{p}$ are called p-paths. By definition, each $p$-path $v \in \Lambda_{p}$ has the form

$$
v=\sum_{i_{0}, \ldots, i_{p} \in V} v^{i_{0} i_{1} \ldots i_{p}} e_{i_{0} i_{1} \ldots i_{p}}, \quad v^{i_{0} i_{1} \ldots i_{p}} \in \mathbb{K} .
$$

For example, 0 -paths are linear combinations of the vertices $e_{i}$ :

$$
v=\sum_{i \in V} v^{i} e_{i}
$$

and 1-paths are linear combinations of pairs of vertices $e_{i j}$ :

$$
v=\sum_{i, j \in V} v^{i j} e_{i j} .
$$

Define the boundary operator $\partial: \Lambda_{p+1} \rightarrow \Lambda_{p}$ by

$$
(\partial v)^{i_{0} \ldots i_{p}}=\sum_{k \in V} \sum_{q=0}^{p+1}(-1)^{q} v^{i_{0} \ldots i_{q-1} k i_{q} \ldots i_{p}}
$$

where the index $k$ is inserted so that it is preceded by $q$ indices. This formula holds for all $p \geqslant 0$. We also need the operator $\partial: \Lambda_{0} \rightarrow \Lambda_{-1}$ where we set $\Lambda_{-1}=\{0\}$ and 
$\partial v=0$ for all $v \in \Lambda_{0}$.

It follows from (2) that

$$
\partial e_{i_{0} \ldots i_{p+1}}=\sum_{q=0}^{p+1}(-1)^{q} e_{i_{0} \ldots \widehat{i_{q}} \ldots i_{p+1}} .
$$

It follows from the definition that, for any $p$-path $v, \partial^{2} v=0$.

An elementary $p$-path $e_{i_{0} \ldots i_{p}}$ is called regular if $i_{k} \neq i_{k+1}$ for all $k$. We would like to define the boundary operator $\partial$ on the subspace of $\Lambda_{p}$ spanned by regular elementary paths. Just the restriction of $\partial$ does not work as $\partial$ is not invariant on this subspace.

Let $I_{p}$ be the subspace of $\Lambda_{p}$ that is spanned by all irregular $e_{i_{0} \ldots i_{p}}$. Consider the quotient space

$$
\mathcal{R}_{p}=\mathcal{R}_{p}(V)=\Lambda_{p} / I_{p} .
$$

The elements of $\mathcal{R}_{p}$ are the equivalence classes $v \bmod I_{p}$, where $v \in \Lambda_{p}$, and they are called regularized $p$-paths. One verifies that the boundary operator $\partial$ is well-defined for regularized paths. Clearly, $\mathcal{R}_{p}$ is linearly isomorphic to the space of regular $p$ paths:

$$
\operatorname{span}\left\{e_{i_{0} \ldots i_{p}}: i_{0} \ldots i_{p} \text { is regular }\right\} .
$$

For simplicity of notation, we will identify $\mathcal{R}_{p}$ with this space by setting all irregular $p$-paths equal to 0 .

Now we define paths on digraphs. A digraph is a pair $G=(V, E)$, where $V$ is an arbitrary set and $E$ is a subset of $V \times V \backslash$ diag. In this paper the set $V$ will be always assumed non-empty and finite. The elements of $V$ are called vertices and the elements of $E$ are called (directed) edges.

The edge starting at a vertex $a$ and ending at $b$ will be denoted by $a b$. The fact that there exists an edge starting at $a$ and ending at $b$ will be denoted by $a \rightarrow b$.

Let $i_{0} \ldots i_{p}$ be a regular elementary $p$-path on $V$. It is called allowed if $i_{k-1} \rightarrow i_{k}$ for any $k=1, \ldots, p$, and non-allowed otherwise.

We would like to reduce the space $\mathcal{R}_{p}$ of regular $p$-paths on $V$ to adapt it to the digraph structure $G$. Denote by $\mathcal{A}_{p}=\mathcal{A}_{p}(G)$ the subspace of $\mathcal{R}_{p}$ spanned by the allowed elementary $p$-paths; that is,

$$
\mathcal{A}_{p}=\operatorname{span}\left\{e_{i_{0} \ldots i_{p}}: i_{0} \ldots i_{p} \text { is allowed }\right\} .
$$

The elements of $\mathcal{A}_{p}$ are called allowed p-paths. Note that $\mathcal{A}_{0}$ consists of linear combination of vertices, and $\mathcal{A}_{1}$ consists of linear combinations of the edges.

In general, the spaces $\mathcal{A}_{p}$ are not invariant for operator $\partial$. For example, if $a b$ and $b c$ are edges then $e_{a b c} \in \mathcal{A}_{2}$, while

$$
\partial e_{a b c}=e_{b c}-e_{a c}+e_{a b}
$$

is non-allowed if $a c$ is not an edge.

Consider the following subspace of $\mathcal{A}_{p}$ :

$$
\Omega_{p}=\Omega_{p}(G)=\left\{v \in \mathcal{A}_{p}: \partial v \in \mathcal{A}_{p-1}\right\} .
$$

Then the family $\left\{\Omega_{p}\right\}$ is $\partial$-invariant. Indeed, if $v \in \Omega_{p}$ then $\partial v \in \mathcal{A}_{p-1}$ and $\partial(\partial v)=$ $0 \in \mathcal{A}_{p-2}$ whence $\partial v \in \Omega_{p-1}$. The elements of $\Omega_{p}$ are called $\partial$-invariant $p$-paths. 
We obtain a chain complex

$$
0 \longleftarrow \Omega_{0} \stackrel{\partial}{\longleftarrow} \Omega_{1} \stackrel{\partial}{\longleftarrow} \quad \cdots \quad \stackrel{\partial}{\longleftarrow} \Omega_{p-1} \stackrel{\partial}{\longleftarrow} \Omega_{p} \stackrel{\partial}{\longleftarrow} \ldots
$$

and the notion of homology groups of the digraph $G$ :

$$
H_{p}(G):=\left.\operatorname{ker} \partial\right|_{\Omega_{p}} /\left.\operatorname{Im} \partial\right|_{\Omega_{p+1}} .
$$

In what follows, we will refer to $H_{p}(G)$ as the graph homologies, in order to distinguish from other theories of homologies.

Now we consider several examples. Let $G=(V, E)$ be a finite digraph. The space $\Omega_{0}$ has always the basis $\left\{e_{a}\right\}_{a \in V}$ and $\Omega_{1}$ has the basis $\left\{e_{a b}\right\}_{a b \in E}$. Let us give examples of $\partial$-invariant paths in $\Omega_{n}$ with $n \geqslant 2$.

Example 3.1. Let us call by a triangle a sequence $\{a, b, c\}$ of three distinct vertices $a, b, c$ of $G$ such that $a b, b c, a c$ are edges:

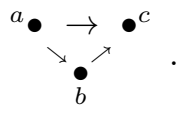

The triangle determines a 2-path $e_{a b c} \in \Omega_{2}$ as $e_{a b c} \in \mathcal{A}_{2}$ and $\partial e_{a b c}=e_{b c}-e_{a c}+e_{a b} \in$ $\mathcal{A}_{1}$. More generally, a graphical $n$-simplex is a sequence $\left\{a_{k}\right\}_{k=0}^{n}$ of $n+1$ distinct vertices from $V$ such that $a_{i} \rightarrow a_{j}$ for all $i<j$. Then $e_{a_{0} \ldots a_{n}}$ and $\partial e_{a_{0} \ldots a_{n}}$ are allowed so that the $n$-path $e_{a_{0} \ldots a_{n}}$ is $\partial$-invariant. One can say that this $n$-path determines the simplex.

Example 3.2. Let us call by a square a sequence $\left\{a, b, b^{\prime}, c\right\}$ of four distinct vertices $a, b, b^{\prime}, c \in V$ such that $a b, b c, a b^{\prime}, b^{\prime} c$ are edges:

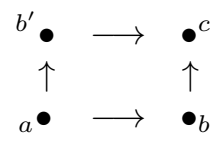

The square determines a 2-path $v=e_{a b c}-e_{a b^{\prime} c} \in \Omega_{2}$ as $v \in \mathcal{A}_{2}$ and

$$
\partial v=\left(e_{b c}-e_{a c}+e_{a b}\right)-\left(e_{b^{\prime} c}-e_{a c}+e_{a b^{\prime}}\right)=e_{a b}+e_{b c}-e_{a b^{\prime}}-e_{b^{\prime} c} \in \mathcal{A}_{1} .
$$

Example 3.3. More generally, a graphical $n$-cube is a set $C$ of $2^{n}$ vertices of $V$ such that any vertex $\alpha \in C$ can be identified with a sequences $\left(\alpha_{1} \ldots \alpha_{n}\right)$ of binary digits so that $\alpha \rightarrow \beta$ if and only if the sequence $\left(\beta_{1} \ldots \beta_{n}\right)$ is obtained from $\left(\alpha_{1} \ldots \alpha_{n}\right)$ by replacing a digit 0 by 1 at exactly one position. The digraph $\bullet \rightarrow \bullet$ is a 1 -cube, a square is a 2-cube, and a 3 -cube is shown in Fig. 3.

With any graphical $n$-cube one can associate a $\partial$-invariant $n$-path as was shown in [9, Example 6.7] (cf. Section 4 below). For example, for the 3-cube as in Fig. 3 this is

$$
v=e_{0457}-e_{0157}+e_{0137}-e_{0467}+e_{0267}-e_{0237} .
$$

It is easy to see that

$$
\begin{aligned}
\partial v= & \left(e_{457}-e_{467}\right)-\left(e_{013}-e_{023}\right)+\left(e_{015}-e_{045}\right) \\
& -\left(e_{237}-e_{267}\right)+\left(e_{137}-e_{157}\right)-\left(e_{026}-e_{046}\right) .
\end{aligned}
$$

In other words, $\partial v$ is an alternating sum of six 2-paths, each of them corresponding to a geometric face of the cube. This observation will be put in a general context in Section 4, and it is a key to the proof of our main Theorem 5.1. 


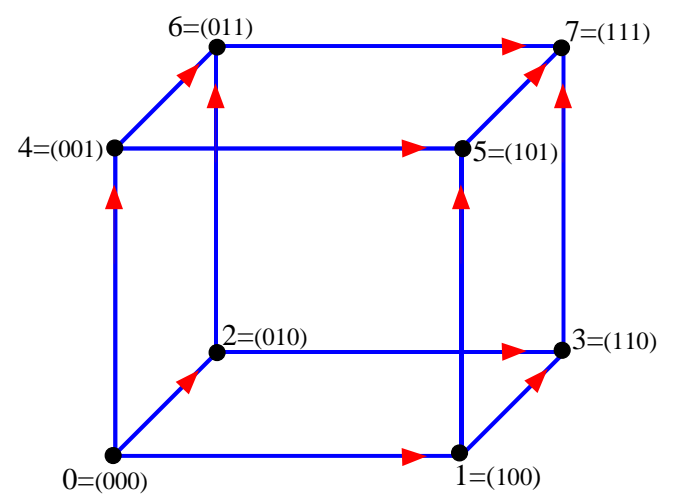

Figure 3: A graphical 3-cube. The binary representations of the vertices are shown in parentheses.

Example 3.4. It is clear that the $\partial$-invariant 2-paths associated to different triangles are linearly independent. Let us give an example showing that the $\partial$-invariant 2-paths associated to different squares can form a linear dependence. Consider the digraph on Fig. 4. It has three squares $\{0,1,2,4\},\{0,1,3,4\},\{0,2,3,4\}$ that give rise to the following three $\partial$-invariant 2-paths

$$
e_{014}-e_{024}, \quad e_{014}-e_{034}, \quad e_{024}-e_{034},
$$

that are obviously linearly dependent. It is possible to show that in this case $\operatorname{dim} \Omega_{2}=$ 2 (cf. [9, Proposition 5.2]).

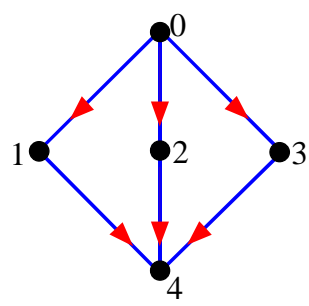

Figure 4: A digraph with linearly dependent squares.

Example 3.5. Consider the (undirected) graph $G$ in Fig. 5 with 6 vertices and 12 edges. As a 1-dimensional simplicial complex, $G$ has simplicial homologies $H_{*}\left(C_{*}(G)\right)$. On the other hand, let us introduce arbitrarily a set $D$ of directions on the edges of $G$, so that $(G, D)$ is a digraph and, hence, has the graph homologies $H_{*}(G, D)$. We claim that for any choice of $D$,

$$
H_{1}\left(C_{*}(G)\right) \neq H_{1}(G, D) \text {. }
$$

As above, let $\left\{\Omega_{n}\right\}$ be the chain complex of the digraph $(G, D)$. In particular, $\operatorname{dim} \Omega_{0}=$ 6 (the number of vertices), and $\operatorname{dim} \Omega_{1}=12$ (the number of edges). By homological 

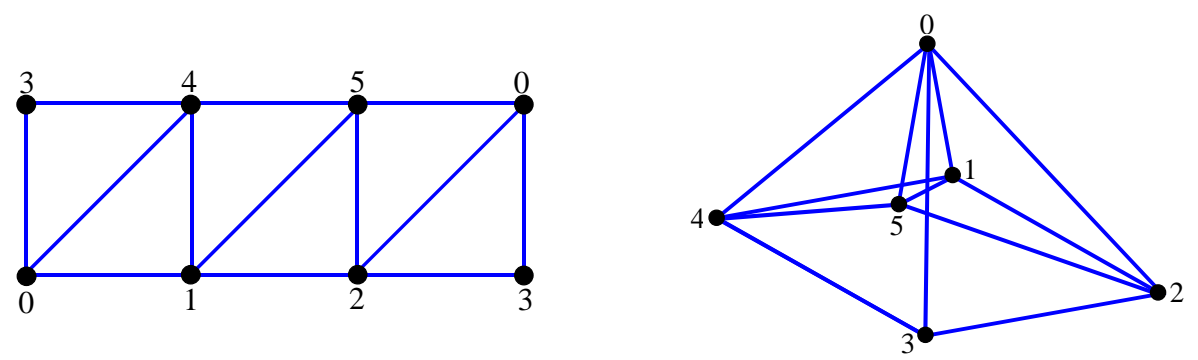

Figure 5: Graph $G$ in two representations: embedded on the Möbius band (left) and in $\mathbb{R}^{3}$ (right). In the left panel, the vertices with the same number are merged.

algebra, we have the following universal identity:

$$
\operatorname{dim} H_{1}(\Omega)-\operatorname{dim} H_{0}(\Omega)=\operatorname{dim} \Omega_{1}-\operatorname{dim} \Omega_{0}-\operatorname{dim} \partial \Omega_{2}
$$

(see, for example, [9, Lemma 3.4]) and an analogous identity for the simplicial homologies. Since the graph $G$ is connected, we have $\operatorname{dim} H_{0}(\Omega)=1$ (cf. [9, Proposition 4.2]). It follows that $\operatorname{dim} H_{1}(\Omega)=7-\operatorname{dim} \partial \Omega_{2}$. A similar formula holds for the simplicial homologies: $\operatorname{dim} H_{1}\left(C_{*}(G)\right)=7-\operatorname{dim} \partial C_{2}(G)$. Since $C_{2}(G)$ is trivial, we obtain $\operatorname{dim} H_{1}\left(C_{*}(G)\right)=7$ (the same can be seen using the homotopy invariance of simplicial homologies as the 1-dimensional simplicial complex $G$ is homotopy equivalent to a wedge sum of seven circles $\left.\mathbb{S}^{1}\right)$.

It remains to show that the space $\partial \Omega_{2}$ is non-trivial for any choice $D$ of the edge directions, which will yield $\operatorname{dim} H_{1}(G, D) \leqslant 6$ and, hence, $(7)$. For that it suffices to verify that there is at least one triangle $\{a, b, c\}$ in $(G, D)$ in the sense of Example 3.1, since then $e_{a b c} \in \Omega_{2}$ and $\partial e_{a b c} \neq 0$. Indeed, let us try to define directions $D$ on the edges of $G$ so that $(G, D)$ contains no triangles. Then any undirected triangle in $G$ must become a cycle

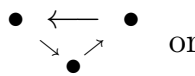

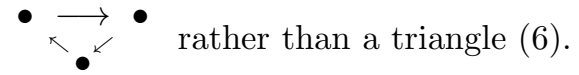

Given a direction of the edge 03, this requirement determines uniquely the directions of all other edges (cf. Fig. 6), up to the edge 23. However, with any direction on 23 the sequence $\{0,2,3\}$ will become a triangle, which finishes the proof.

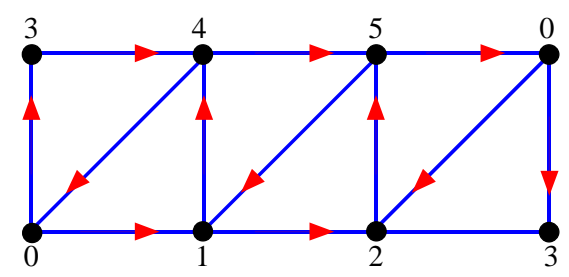

Figure 6: An attempt to introduce on $G$ the direction of edges. Any direction of the edge 23 will create a triangle. 


\section{Cubical digraphs}

Let $M$ be a finite set with $m$ elements. Let us introduce in the power set $2^{M}$ of $M$ the structure of a digraph as follows: for two arbitrary sets $s_{1}, s_{2} \in 2^{M}$, define the edge between them by the rule

$$
s_{1} \rightarrow s_{2} \Leftrightarrow s_{2} \text { is obtained from } s_{1} \text { by removing of exactly one element. }
$$

Denote this digraph by $G_{M}$. Let us fix an enumeration of the elements of $M$ by integers $0,1, \ldots, m-1$; in fact, identify $M$ with the set $\{0,1, \ldots, m-1\}$. For any set $s \in 2^{M}$ define its anti-indicator $N(s)$ by

$$
N(s)=\sum_{i \in M \backslash s} 2^{i} .
$$

For example, $N(\emptyset)=2^{m}-1$ and $N(M)=0$. Clearly, if $s_{1} \rightarrow s_{2}$ then

$$
N\left(s_{2}\right)=N\left(s_{1}\right)+2^{i},
$$

where $i$ is the unique element in $s_{1} \backslash s_{2}$.

Let $S$ be a family of subsets of $M$; that is, $S \subset 2^{M}$. Denote by $G_{S, M}$ the digraph with the vertex set $S$, whose edges are all the edges from $G_{M}$ with the endpoints in $S$. If no confusion arises, we write the shorthand $G_{S}$ instead of $G_{S, M}$.

Definition 4.1. The digraph $G_{S}$ is called cubical if the family $S \subset 2^{M}$ possesses the following property: if $s, t$ are two elements of $S$, then any subset $u$ of $M$ such that $s \subset u \subset t$ is also an element of $S$.

For example, the digraph $G_{M}\left(S=2^{M}\right)$ is a cubical graph. The reason for the term "cubical" is that $G_{M}$ is, in fact, a graphical $m$-cube. Indeed, with each element $s \in$ $2^{M}$ consider $N(s)$ as a binary number, which provides a one-to-one correspondence between $2^{M}$ and the sequences of $m$ binary digits. Moreover, $s_{1} \rightarrow s_{2}$ means by (9) that $N\left(s_{2}\right)$ is obtained from $N\left(s_{1}\right)$ by replacing one binary digit 0 by 1 . Hence, $G_{M}$ is a graphical $m$-cube (cf. Fig. 7). In fact, $G_{M}$ is nothing other than the inverted Hasse diagram of the partially ordered set $2^{M}$.
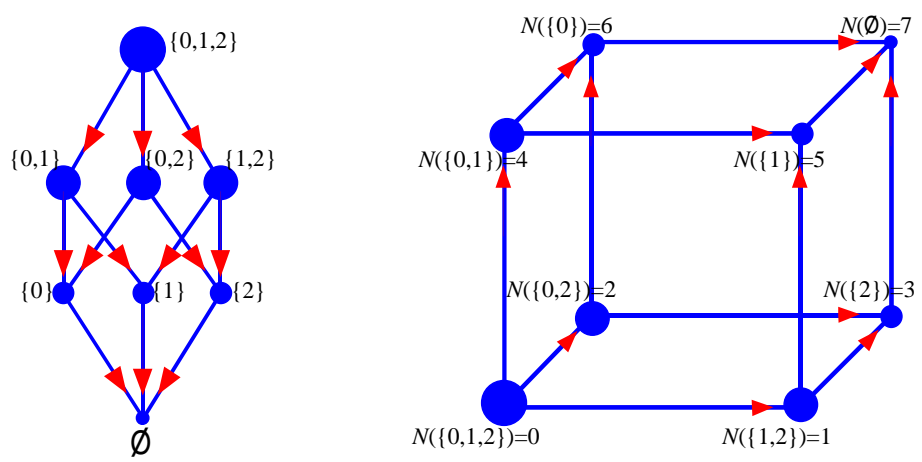

Figure 7: The cubical graph $G_{M}$ for $M=\{0,1,2\}$ drawn in two ways. In the right panel, each vertex $s$ is assigned the number $N(s)$. 
Example 4.2. With any simplicial complex $S$ we associate a cubical digraph as follows. Denote by $M$ the set of all vertices of $S$ (with a fixed enumeration as above). Then any $k$-simplex in $S$ can be regarded as a $(k+1)$-subset of $M$, and $S$ can be regarded as a subset of $2^{M}$. By the above construction, we obtain a digraph $G_{S}$. It satisfies the definition of a cubical graph because by definition of a simplicial complex, if a subset $s$ of $M$ is a simplex from $S$, then any non-empty subset $s^{\prime}$ of $s$ is also a simplex of $S$.

Equivalently, one can describe the graph $G_{S}$ of a simplicial complex $S$ as follows. The set of vertices of $G_{S}$ coincides with the set of all simplexes from $S$. The edges in $G_{S}$ are defined by (8) or, equivalently, by

$$
s \rightarrow t \Leftrightarrow s \supset t \text { and } \operatorname{dim} s=\operatorname{dim} t+1,
$$

where $s, t$ are simplexes from $S$ (cf. Fig. 1 in Introduction).

Now we describe properties of general cubical digraphs that provide an effective tool for computing homologies.

Fix a set $M=\{0,1, \ldots, m-1\}$ as above, and consider the digraph $G_{M}$. Let $\left\{\alpha_{k}\right\}_{k=0}^{n}$ be an allowed path in $G_{M}$; that is, $\alpha_{k-1} \rightarrow \alpha_{k}$ for all $k=1, \ldots, n$. Define a non-negative integer $\sigma(\alpha)$ as follows. Since $\alpha_{k-1} \rightarrow \alpha_{k}$, there is a unique value $i_{k} \in\{0,1, \ldots, m-1\}$ such that

$$
\alpha_{k-1} \backslash \alpha_{k}=\left\{i_{k}\right\}
$$

or, equivalently,

$$
N\left(\alpha_{k}\right)=N\left(\alpha_{k-1}\right)+2^{i_{k}} .
$$

Then define $\sigma(\alpha)$ as the number of inversions in the sequence $\left\{i_{1}, \ldots, i_{n}\right\}$ (cf. Fig. 8).

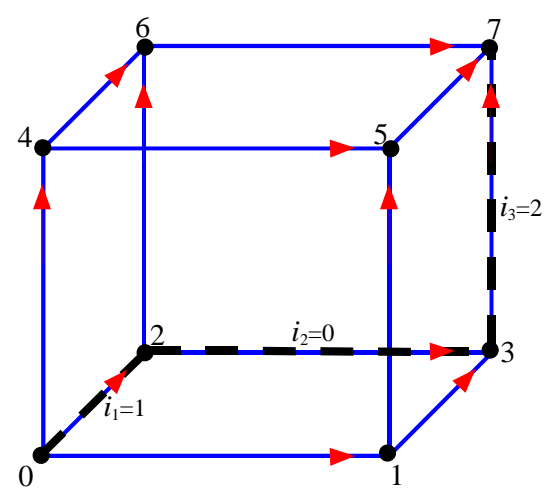

Figure 8: For the path $\alpha=0237$, the sequence $\left\{i_{1}, i_{2}, i_{3}\right\}$ is $\{1,0,2\}$, and it has one inversion. Hence, $\sigma(\alpha)=1$.

Lemma 4.3. Let $\alpha=\left\{\alpha_{k}\right\}_{k=0}^{n}$ be an allowed path in $G_{M}$.

(a) Denote by $\alpha^{\prime}$ the truncated sequence $\left\{\alpha_{k}\right\}_{k=1}^{n}$ so that $\alpha^{\prime}$ is an allowed path. Then the difference $\sigma(\alpha)-\sigma\left(\alpha^{\prime}\right)$ depends only on $\alpha_{0}, \alpha_{1}, \alpha_{n}$. 
(b) Denote by $\alpha^{\prime}$ the truncated sequence $\left\{\alpha_{k}\right\}_{k=0}^{n-1}$ so that $\alpha^{\prime}$ is an allowed path. Then the difference $\sigma(\alpha)-\sigma\left(\alpha^{\prime}\right)$ depends only on $\alpha_{0}, \alpha_{n-1}, \alpha_{n}$.

Proof. Indeed, let $i_{k}$ be as in (11). Then $\sigma(\alpha)$ is the number of inversions in the sequence $\left\{i_{1}, i_{2}, \ldots, i_{n}\right\}$ while $\sigma\left(\alpha^{\prime}\right)$ is the number of inversions in the sequence $\left\{i_{2}, i_{3}, \ldots, i_{n}\right\}$. Therefore, the difference $\sigma(\alpha)-\sigma\left(\alpha^{\prime}\right)$ is the number of inversions of $i_{1}$ in $\left\{i_{1}, i_{2}, \ldots, i_{n}\right\}$; that is, the number of the values $i_{2}, \ldots, i_{n}$ that are smaller than $i_{1}$. Since by (11)

$$
N\left(\alpha_{n}\right)-N\left(\alpha_{1}\right)=2^{i_{2}}+2^{i_{3}}+\cdots+2^{i_{n}},
$$

and all $i_{k}$ are different, the values of $i_{2}, \ldots, i_{n}$ (but not the order) are uniquely determined by $N\left(\alpha_{n}\right)-N\left(\alpha_{1}\right)$. Since $i_{1}$ is determined by $N\left(\alpha_{1}\right)-N\left(\alpha_{0}\right)$, the number of the values $i_{2}, \ldots, i_{n}$ that are smaller than $i_{1}$ is determined by $N\left(\alpha_{n}\right)-N\left(\alpha_{1}\right)$ and $N\left(\alpha_{1}\right)-N\left(\alpha_{0}\right)$, which finishes the proof of $(a)$. Part $(b)$ is proved similarly.

For any two subsets $s, t$ of $M$, such that $t \subset s$, denote by $D_{s, t}$ the family of all subsets $u \subset M$ such that $t \subset u \subset s$. We consider $D_{s, t}$ as a digraph with the edges as in (8). Clearly, $D_{s, t}$ is a subgraph of $G_{M}$ and $D_{s, t}$ is isomorphic to the digraph $G_{s \backslash t}$ so that $D_{s, t}$ is a graphical $n$-cube, where $n=|s|-|t|$. Note that if $S \subset 2^{M}$ satisfies the property of Definition 4.1 and $s, t$ are two elements of $S$ such that $t \subset s$, then $D_{s, t}$ is a subgraph of $S$.

For any $n$-cube $D_{s, t} \subset G_{M}$ denote by $P\left(D_{s, t}\right)$ the set of all allowed paths $\left\{\alpha_{k}\right\}_{k=0}^{n}$ such that $\alpha_{0}=s$ and $\alpha_{n}=t$. Then $t \subset \alpha_{k} \subset s$ for any $k$, so that all $\alpha_{k}$ belong to $D_{s, t}$. Any path $\alpha \in P\left(D_{s, t}\right)$ is called a full chain in $D_{s, t}$. With each $n$-cube $D=D_{s, t}$ let us associate a $n$-path $\omega=\omega(D)$ by

$$
\omega(D)=\sum_{\alpha \in P(D)}(-1)^{\sigma(\alpha)} e_{\alpha} .
$$

Since each $n$-path $e_{\alpha}=e_{\alpha_{0} \ldots \alpha_{n}}$ is allowed in $D$, the $n$-path $\omega(D)$ is also allowed. We will show below that $\omega(D)$ is, in fact, $\partial$-invariant in $D$.

Let $D=D_{s, t}$ be an $n$-cube in $G_{M}$. For any $(n-1)$-cube $D^{\prime} \subset D$ define the number $\sigma\left(D, D^{\prime}\right)$ as follows. For $D^{\prime}$ there are two possibilities:

1. either $D^{\prime}=D_{s^{\prime}, t}$ where $s \rightarrow s^{\prime}$;

2. or $D^{\prime}=D_{s, t^{\prime}}$ where $t^{\prime} \rightarrow t$.

In the first case consider any full chain $\alpha \in P(D)$ with $\alpha_{1}=s^{\prime}$ and set $\alpha^{\prime}=$ $\left\{\alpha_{k}\right\}_{k=1}^{n}$ so that $\alpha^{\prime} \in P\left(D^{\prime}\right)$. Then define

$$
\sigma\left(D, D^{\prime}\right)=\sigma(\alpha)-\sigma\left(\alpha^{\prime}\right) .
$$

In the second case consider a full chain $\alpha \in P(D)$ with $\alpha_{n-1}=t^{\prime}$ and set $\alpha^{\prime}=$ $\left\{\alpha_{k}\right\}_{k=0}^{n-1}$ so that $\alpha^{\prime} \in P\left(D^{\prime}\right)$. Then define

$$
\sigma\left(D, D^{\prime}\right)=(-1)^{n}\left(\sigma(\alpha)-\sigma\left(\alpha^{\prime}\right)\right) .
$$

Note that by Lemma 4.3 the value of $\sigma\left(D, D^{\prime}\right)$ in the both cases does not depend on the choice of $\alpha$ : in the first case $\sigma\left(D, D^{\prime}\right)$ depends on $s, s^{\prime}, t$; in the second case, on $s, t^{\prime}, t$. 
Lemma 4.4. For any $n$-cube $D$ in $G_{M}$ we have

$$
\partial \omega(D)=\sum_{D^{\prime} \subset D}(-1)^{\sigma\left(D, D^{\prime}\right)} \omega\left(D^{\prime}\right),
$$

where the sum is taken over all $(n-1)$-cubes $D^{\prime} \subset D$. Consequently, $\omega(D)$ is a $\partial$ invariant path in the digraph $D$.

Proof. We have

$$
\begin{aligned}
\partial \omega= & \sum_{\alpha}(-1)^{\sigma(\alpha)} \partial e_{\alpha_{0} \alpha_{1} \ldots \alpha_{n}} \\
= & \sum_{\alpha}(-1)^{\sigma(\alpha)} \sum_{k=0}^{n}(-1)^{k} e_{\alpha_{0} \ldots \widehat{\alpha_{k}} \ldots \alpha_{n}} \\
= & \sum_{\alpha}(-1)^{\sigma(\alpha)} e_{\alpha_{1} \ldots \alpha_{n}}+(-1)^{n} \sum_{\alpha}(-1)^{\sigma(\alpha)} e_{\alpha_{0} \ldots \alpha_{n-1}} \\
& +\sum_{k=1}^{n-1}(-1)^{k} \sum_{\alpha}(-1)^{\sigma(\alpha)} e_{\alpha_{0} \ldots \widehat{\alpha_{k}} \ldots \alpha_{n}} .
\end{aligned}
$$

Observe that for any $k=1, \ldots, n-1$

$$
\sum_{\alpha}(-1)^{\sigma(\alpha)} e_{\alpha_{0} \ldots \widehat{\alpha_{k}} \ldots \alpha_{n}}=0 .
$$

Indeed, it suffices to show that

$$
\sum_{\alpha_{k}}(-1)^{\sigma(\alpha)} e_{\alpha_{0} \ldots \widehat{\alpha_{k}} \ldots \alpha_{n}}=0 .
$$

Since $\alpha_{k-1}$ and $\alpha_{k+1}$ are fixed, for $\alpha_{k}$ there are only two possibilities, and $\sigma(\alpha)$ for these two possibilities have different parity, so that the term $e_{\alpha_{0} \ldots \widehat{\alpha_{k}} \ldots \alpha_{n}}$ cancels out.

Denoting by $s^{\prime}$ any successor of $s$ and by $t^{\prime}$ any predecessor of $t$, we obtain

$$
\begin{aligned}
\partial \omega & =\sum_{\alpha}(-1)^{\sigma(\alpha)} e_{\alpha_{1} \ldots \alpha_{n}}+(-1)^{n} \sum_{\alpha}(-1)^{\sigma(\alpha)} e_{\alpha_{0} \ldots \alpha_{n-1}} \\
& =\sum_{s^{\prime}} \sum_{\alpha: \alpha_{1}=s^{\prime}}(-1)^{\sigma(\alpha)} e_{\alpha_{1} \ldots \alpha_{n}}+(-1)^{n} \sum_{t^{\prime}} \sum_{\alpha: \alpha_{n-1}=t^{\prime}}(-1)^{\sigma(\alpha)} e_{\alpha_{0} \ldots \alpha_{n-1}} .
\end{aligned}
$$

The sequence $\alpha_{1} \ldots \alpha_{n}$ with $\alpha_{1}=s^{\prime}$ and $\alpha_{n}=t$ determines a $(n-1)$-subcube $D^{\prime}=$ $D_{s^{\prime}, t}$ of $D_{s, t}$. Denoting $\alpha^{\prime}=\alpha_{1} \ldots \alpha_{n}$ (that is, a full chain of $D_{s^{\prime}, t}$ ), we obtain

$$
\begin{aligned}
\sum_{\alpha: \alpha_{1}=s^{\prime}}(-1)^{\sigma(\alpha)} e_{\alpha_{1} \ldots \alpha_{n}} & =\sum_{\alpha^{\prime} \in P\left(D^{\prime}\right)}(-1)^{\sigma(\alpha)} e_{\alpha_{1}^{\prime} \ldots \alpha_{n}^{\prime}} \\
& =\sum_{\alpha^{\prime} \in P\left(D^{\prime}\right)}(-1)^{\sigma(\alpha)-\sigma\left(\alpha^{\prime}\right)}(-1)^{\sigma\left(\alpha^{\prime}\right)} e_{\alpha_{1}^{\prime} \ldots \alpha_{n}^{\prime}} \\
& =(-1)^{\sigma\left(D, D^{\prime}\right)} \omega\left(D^{\prime}\right),
\end{aligned}
$$

where we have used (13). Hence,

$$
\sum_{\alpha}(-1)^{\sigma(\alpha)} e_{\alpha_{1} \ldots \alpha_{n}}=\sum_{D^{\prime} \subset D}(-1)^{\sigma\left(D, D^{\prime}\right)} \omega\left(D^{\prime}\right),
$$


where the summation extends to all $(n-1)$-cubes $D^{\prime} \subset D$ with the same target $t$.

Similarly, a sequence $\alpha_{0} \ldots \alpha_{n-1}$ with $\alpha_{n-1}=t^{\prime}$ determines a $(n-1)$-subcube $D^{\prime}=D_{s, t^{\prime}}$ of $D_{s, t}$. Denoting $\alpha^{\prime}=\alpha_{0} \ldots \alpha_{n-1}$, we obtain

$$
(-1)^{n} \sum_{\alpha^{\prime} \in P\left(D^{\prime}\right)}(-1)^{\sigma(\alpha)} e_{\alpha_{0}^{\prime} \ldots \alpha_{n-1}^{\prime}}=(-1)^{\sigma\left(D, D^{\prime}\right)} \omega\left(D^{\prime}\right)
$$

where we have used (14). Therefore,

$$
(-1)^{n} \sum_{\alpha}(-1)^{\sigma(\alpha)} e_{\alpha_{0} \ldots \alpha_{n-1}}=\sum_{D^{\prime} \subset D}(-1)^{\sigma\left(D, D^{\prime}\right)} \omega\left(D^{\prime}\right),
$$

where the summation extends to all $(n-1)$-cubes $D^{\prime} \subset D$ with the same source $s$. Combining together (16) and (17) we obtain (15).

Finally, since all $\omega\left(D^{\prime}\right)$ are allowed paths in $D$, we obtain that $\partial \omega(D)$ is allowed and, hence, $\omega$ is $\partial$-invariant.

Let $G_{S}$ be a cubical digraph based in a set $M$. Consider a digraph $T_{S}$ with the same set of vertices and with the following set of edges. For any $n$-cube $D_{s, t} \subset G_{S}$ and $s \supset u \supset t$, the edges $s \rightarrow u(u \neq s)$ and $u \rightarrow t(u \neq t)$ lay in $T_{S}$. It is clear that we have an inclusion $G_{S} \rightarrow T_{S}$ of digraphs; that is, an identity map on the set of vertices. It follows immediately from this definition that any admissible path in $T_{S}$ is $\partial$-invariant; that is, $\Omega_{p}\left(T_{S}\right)=\mathcal{A}_{p}\left(T_{S}\right), p \geqslant 0$.

Now we define a topological realization $Q_{S}$ of the cubical digraph $G_{S}$ as a cubical cell complex and define a natural simplicial subdivision $\Delta_{S}$ of $G_{S}$. Let $Q_{S}$ be a cubical complex in which the cubical $n$-cells $q_{s, t}$ are in one-to-one correspondence with the cubes $D_{s, t} \in G_{S}$ and the incidence relation is induced from incidence relation in $G_{S}$. To any full chain $\alpha=\left\{\alpha_{k}\right\}_{k=0}^{n} \in P\left(D_{s, t}\right)\left(\alpha_{0}=s, \alpha_{n}=t\right)$ we assign a $n$-simplex $\tau_{\alpha}$ given by the set of his vertices $\tau_{\alpha}=\left\{b_{\alpha_{k}}\right\}_{k=0}^{n}$. Thus, in particular, we identify the vertices of $G_{S}$ with 0 -cells of $Q_{S}$. The cell $q_{s, t}$ is a union

$$
q_{s, t}=\bigcup_{\alpha \in P\left(D_{s, t}\right)} \tau_{\alpha}
$$

and hence the simplexes $\tau_{\alpha}$ with $\alpha \in P\left(D_{s, t}\right)$ give a standard simplicial subdivision of the cubical cell $q_{s, t}$. We denote the obtained simplicial complex by $\Delta_{S}$. It follows from the definition that

$$
H_{*}\left(\left|Q_{S}\right|\right) \cong H_{*}\left(\left|\Delta_{S}\right|\right) \cong H_{*}\left(C_{*}\left(\Delta_{S}\right)\right),
$$

where $H_{*}\left(C_{*}\left(\Delta_{S}\right)\right)$ is the simplicial chain complex of $\Delta_{S}$. Additionally, we have an isomorphism of chain complexes

$$
i: \Omega_{*}\left(T_{S}\right) \cong C_{*}\left(\Delta_{S}\right),
$$

given on the set of admissible paths by $i(\beta)=\tau_{\beta}$, where $\beta=\beta_{0} \rightarrow \beta_{1} \rightarrow \cdots \rightarrow \beta_{k}$ is an admissible path in $T_{S}$ and the simplex $\tau_{\beta}$ is given by the set of his vertices $\left(\beta_{0}, \beta_{1}, \ldots, \beta_{k}\right)$. Hence we have an isomorphism $H_{*}\left(T_{S}\right) \cong H_{*}\left(C\left(\Delta_{S}\right)\right)$. 


\section{Homology of cubical digraphs}

Now the following theorem and its corollary give the main results of this paper stated in the Introduction. All homologies are considered over a fixed ring $\mathbb{K}$.

Theorem 5.1. Let $G_{S}$ be a cubical digraph based in a set $M$ and $K_{n}$ be the number of $n$-cubes that are contained in the digraph $G_{S}$. Then $\operatorname{dim} \Omega_{n}(S)=K_{n}$ and for $n \geqslant 0$ we have an isomorphism $H_{n}\left(G_{S}\right) \cong H_{n}\left(C_{*}\left(\Delta_{S}\right)\right)$.

Remark 5.2. This statement is not true for a general digraph. Although any $n$-cube $D$ in an arbitrary digraph always gives rise to the $\partial$-invariant $n$-path $\omega(D)$, as in Lemma 4.4, the paths $\omega(D)$ associated with different cubes $D$ can be linearly dependent as was shown in Example 3.4.

Proof. Consider a cubical complex $Q_{S}$ as above and his simplicial subdivision $\Delta_{S}$ given by (18). Define orientations of the cubes in $Q_{S}$, taking the orientation of $q_{s, t}$ that coincides with the orientation of the $n$-dimensional simplex $\tau_{\alpha} \subset q_{s, t}$ of the subdivision $T_{S}$ for which the sequence of indexes $\left(i_{1}=\alpha_{0} \backslash \alpha_{1}, \ldots, i_{n}=\alpha_{n-1} \backslash \alpha_{n}\right)$ is in increasing order. We equip every simplex $\tau_{\alpha^{\prime}}$ in decomposition (18) with an orientation that is given by the order of its vertices in the path $\alpha^{\prime}$.

We have the natural injective homomorphism of cellular chain complexes (see, for example, [10, Sec. 3.8])

$$
j: C_{*}\left(Q_{S}\right) \rightarrow C_{*}\left(T_{S}\right), \quad j\left(q_{s, t}\right)=\sum_{\alpha \in P(D)}(-1)^{\sigma(\alpha)} \tau_{\alpha} .
$$

Denote by $F_{n} \subset C_{n}\left(T_{S}\right)$ the subgroup generated by all $n$-simplexes of $T_{S}$ that lie in $n$-cells $q_{s, t} \in Q_{S}$; that is, $F_{n}$ is generated by all simplexes $\tau_{\alpha}$ where $\alpha=\left\{\alpha_{k}\right\}_{k=0}^{n}$ is an allowed path in $D_{s, t} \subset G_{S}$. Let $A_{n}$ be a subgroup of $F_{n}$, consisting of all chains $c \in F_{n}$ such that $\partial c \in F_{n-1}$.

Lemma 5.3. There is an isomorphism $j\left(C_{n}\left(Q_{S}\right)\right)=A_{n}$.

Proof. It follows from (21) and (18) for a cube $q_{s, t} \in C_{n}\left(Q_{S}\right)$ that $j\left(q_{s, t}\right) \in A_{n}$, since the map $j$ is a chain map. Hence $j\left(C_{n}\left(Q_{S}\right)\right) \subset A_{n}$.

Now we prove an inverse inclusion $A_{n} \subset j\left(C_{n}\left(Q_{S}\right)\right)$. Consider a chain

$$
f_{n}=\sum_{\alpha \in P} k_{\alpha} \tau_{\alpha} \in F_{n}, \quad k \in \mathbb{K}, P=\left\{P\left(D_{s, t}\right):|s \backslash t|=n\right\}
$$

such that $\partial f_{n} \in F_{n-1}$. It is sufficient to consider the case where all simplexes $\tau_{\alpha}$ lie in one cube $q_{s, t}$, and then use an induction by the number of cubes.

Thus, let $q=q_{s, t}$ be an $n$-cube, $P=P\left(D_{s, t}\right)$, and

$$
f_{n}=\sum_{\alpha \in P} k_{\alpha} \tau_{\alpha} \in F_{n}, \quad k \in \mathbb{K}, \partial f_{n} \in F_{n-1} .
$$

Take any simplex $\tau_{\alpha}=\left\{b_{\alpha_{0}}, \ldots, b_{\alpha_{n}}\right\}$ fitting in the sum with a nonzero coefficient and consider its boundary. Only two simplexes

$$
\left\{b_{\alpha_{1}}, \ldots, b_{\alpha_{n}}\right\}, \quad\left\{b_{\alpha_{0}}, \ldots, b_{\alpha_{n-1}}\right\}
$$

from its boundary lie in $F_{n-1}$. Hence for any another simplex of the boundary $\partial\left(\tau_{\alpha}\right)$, 
say $\partial_{k}\left(\tau_{\alpha}\right)=\left\{b_{\alpha_{0}}, \ldots, \widehat{b_{\alpha_{k}}}, \ldots, b_{\alpha_{n}}\right\}$, there exists only one simplex $\tau_{\alpha^{\prime}} \in D_{s, t}$, where

$$
\alpha_{i}^{\prime}= \begin{cases}\alpha_{i}, & i \neq k \\ \alpha_{k-1} \backslash\left\{i_{k+1}\right\}, & \text { where } i_{k+1}=\alpha_{k} \backslash \alpha_{k+1}\end{cases}
$$

for which the boundary contains the simplex $\partial_{k}\left(\tau_{\alpha}\right)$ with the opposite orientation.

Hence the simplex $\tau_{\alpha^{\prime}}$ fits in the sum (22) with the coefficient $k_{\alpha^{\prime}}=-k_{\alpha}$. Extending this process and using (21) we obtain that the sum in (22) coincides with $\pm k_{\alpha} j\left(q_{s, t}\right)$. This finishes the proof of the lemma.

Now consider a diagram

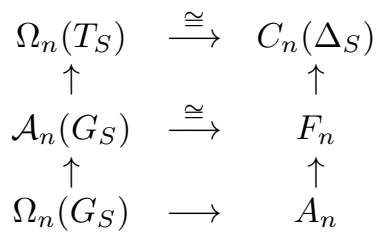

where $A_{n}=j\left(C_{n}\left(Q_{S}\right)\right) \cong C_{n}\left(Q_{S}\right)$ by Lemma 5.3. The vertical maps in the diagram are inclusions, the two upper horizontal maps are isomorphisms since they are restrictions of the isomorphism $i$ (20), and the upper square is commutative. The map $i$ is a morphism of chain complexes and the subcomplexes $\Omega_{n}\left(G_{S}\right) \subset \mathcal{A}_{n}\left(G_{S}\right), A_{n} \subset F_{n}$ in bottom row are defined by the same condition to be $\partial$-invariant. Hence the diagram is commutative, and the bottom horizontal map is an isomorphism. Now the statement of the theorem follows.

\section{References}

[1] B. Chen, S.-T. Yau, and Y.-N. Yeh, Graph homotopy and Graham homotopy, Discrete Math. 241 (2001), 153-170.

[2] A. Dimakis and F. Müller-Hoissen, Differential calculus and gauge theory on finite sets, J. Phys. A, Math. Gen. 27 (1994), 3159-3178.

[3] A. Dimakis and F. Müller-Hoissen, Discrete differential calculus: Graphs, topologies, and gauge theory, J. Math. Phys. 35 (1994), 6703-6735.

[4] N.P. Dolbilin, Yu. M. Zinoviev, A.S. Mishchenko, M.A. Shtan'ko, and M.I. Shtogrin, Homological Properties of Dimer Configurations for Lattices on Surfaces, Funct. Anal. Appl 30 (1996), 163-173.

[5] N.P. Dolbilin, M.A. Shtan'ko, and M.I. Shtogrin, Quadrillages and parametrizations of lattice cycles, Proc. Steklov Inst. Math. 196 (1991), 73-93.

[6] N.P. Dolbilin, M.A. Shtan'ko, and M.I. Shtogrin, Cubic manifolds in lattices, Izv. Ross. Akad. Nauk Ser. Mat. 58 (1994), 93-107.

[7] A.V. Ivashchenko, Contractible transformations do not change the homology groups of graphs, Discrete Math. 126 (1994), 159-170.

[8] A. Grigor'yan and Y. Muranov, Differential calculus on algebras and graphs, preprint, 2012. 
[9] A. Grigor'yan, Y. Lin, Y. Muranov, and S.-T. Yau, Homologies of path complexes and digraphs, preprint, 2012.

[10] P.J. Hilton and S. Wylie, Homology theory, University Press, Cambridge, 1960.

[11] S. MacLane, Homology, Springer-Verlag, Berlin-Göttingen-Heidelberg, 1963.

[12] V.V. Prasolov, Elements of homology theory, Graduate Studies in Mathematics 81, AMS, Providence, 2007.

[13] M.A. Shtan'ko and M.I. Shtogrin, Embedding cubic manifolds and complexes into a cubic lattice, Russian Math. Surveys 47 (1992), 267-268.

A. Grigor'yan grigor@math.uni-bielefeld.de

Mathematics Department, University of Bielefeld, Postfach 100131, D-33501 Bielefeld, Germany

Yu.V. Muranov ymuranov@mail.ru

Mathematics Department, Grodno State University, Ozheshko str. 22, Grodno 230023, Belarus

Shing-Tung Yau yau@math.harvard.edu

Mathematics Department, Harvard University, One Oxford Street, Cambridge MA 02138, USA 